\section{Interactions with Diagrams and the Making of Reasoned Conjectures in Geometry}

\author{
Patricio Herbst, ${ }^{1}$ Ann Arbor (USA)
}

\begin{abstract}
:
Four potential modes of interaction with diagrams in geometry are introduced. These are used to discuss how interaction with diagrams has supported the customary work of 'doing proofs' in American geometry classes and what interaction with diagrams might support the work of building reasoned conjectures. The extent to which the latter kind of interaction may induce tensions on the work of a teacher as she manages students' mathematical work is illustrated.
\end{abstract}

\section{Kurzreferat:}

Vier mögliche Formen der Interaktion mit geometrischen Darstellungen werden aufgezeigt. Diese Formen werden thematisiert um deutlich zu machen, wie visuelle Darbietungen im amerikanischen Geometrieunterricht das alltägliche Geschäft des Beweisens, unterstützen. Dadurch soll auch gezeigt werden, welche Art der Interaktion mit geometrischen Darstellungen es erlaubt, das Herstellen begründeter Vermutungen $\mathrm{zu}$ unterstützen. Zugleich wird das Ausmaß illustriert, mit welchem die letztere Art von Interaktion Spannungen innerhalb der unterrichtlichen Arbeit, der Lehrerin hervorruft, die sich darum bemüht, die mathematischen Beiträge, d.h. die mathematische Arbeit, der Schülerinnen und Schüler zu organisieren.

ZDM-Classification: C63, C73, D43, E 53, G43

\section{Introduction}

Conjecturing and proving are part of a natural, cognitive unity in mathematics, one that revolves around the activity of producing theorems, often in response to problems (Mariotti et al. 1997). Mathematical proof plays an essential role in bringing theorems into existence, in shaping reasonable conjectures and building a reasoned discourse. Lakatos's (1976) analysis of the emergence of mathematical ideas through its historical production by the mathematical community (and in contrast to the impression created by the usual a-historical, decontextualized presentation of ideas in textbooks), shows how proof is intrinsically tied to the development (and not just the justification) of knowledge: Proving is a tool for shaping private intuitions into reasonable assertions about concepts as these are publicly developed. As Lakatos (1976) argues, the dialectic of informal proofs and refutations is key in improving a naïve conjecture and achieving a theorem. Proving is instrumental to conjecturing - not least because, to the extent that proofs and refutations also shape mathematical concepts (making them into abstractions), proving is the tool that best can handle them. The extent to which a proof needs to be formal to be also effective depends quite a bit on the extent to which concepts need to be detached from informal systems of representation for those who handle them to accept those concepts as being well defined. And inasmuch as the proofs that serve mathematical creation are, more often than not, informal proofs, one can also say that conjecturing is rarely like wild guessing. As Lakatos argues, the heuristic reasoning that leads to the production of mathematical knowledge can better accommodate conjecturing as "deductive guessing."

I refer to this aspect of authentic mathematical activity, that dynamic relationship between what is known and how it is known, as building reasoned conjectures. A question with which mathematics educators need to contend is whether the building of reasoned conjectures can be sustained in classrooms (see Chazan, 1995). Lakatos' argument is very relevant as we seek to envision the place that reasoning and proving could have in mathematics classrooms. The notion that proving is an effective way of handling mathematical objects, adapted to the level of abstraction of those objects and to the resources and demands of the theoretical problematiques for which they are needed, seems like a useful, instrumental way of thinking about proof (although if anything it provides an unconventional answer to the more philosophical question of what a proof is).

But the question of what can be done in classrooms is not only a question about the design of interventions or policies, but also one of "ecological" viability of ideas and practices in contexts that are institutional as much as they are interpersonal and disciplinary. Ideas and practices can exist only if they are viable within the conditions and constraints of a particular context. Is it feasible for teachers to engage students in building reasoned conjectures, and what does it take for a teacher to do that? The high school geometry class in the United States is an appropriate context for investigating such question. This class has historically made room for conjecture and proof, and in general has been billed as students' first encounter with theoretical mathematics (Moise 1975; Usiskin 1980).

This paper contributes to the goal of understanding what conditions enable students to engage in building reasoned conjectures by looking at the role of students' interaction with geometric diagrams in classroom situations that involve students in conjecturing and in proving. Specifically, I propose a distinction between modes in which students' interaction with diagrams might support the work of conjecturing, doing proofs, and building reasoned conjectures. Then I use those modes of interaction to describe the kinds of conjecturing and proving that ordinarily take place in the high school geometry class. I argue that those modes of interaction are part of larger accountability structures, or situations, that establish who-has-to-do-what-to-get-what in various classroom situations. And I then argue that situations that would aim at students' making of reasonable conjectures in geometry require interactions with diagrams that breach those norms.

\section{Modes of interaction with diagrams}

The purpose of this section is to provide some language for thinking about the possible relationships between a 
person, a diagram, and geometric objects. Through the article I suggest four possible modes in which students' interaction with a diagram, and their discourse about the geometric objects at stake in the diagram, may relate. Each of the four modes of interaction (which I term empirical, representational, descriptive, and generative) instantiates a set of relationships between three elements: a subject or actor (A), a physical diagram or representation (D), and a theoretical, geometric object, at stake in that interaction $(\mathrm{O})$. Another, compelling way of describing an individual's relationship with figures has been proposed by Duval (1995), who speaks of different kinds of cognitive "apprehensions" of figures. Duval makes the important point that for a picture to act as a geometric diagram it needs to be apprehended perceptually (as a graphic object in contrast with its background) and at least in one other way-sequentially, discursively, or operatively. I comment on those other apprehensions as I relate Duval's categories to the proposed ones.

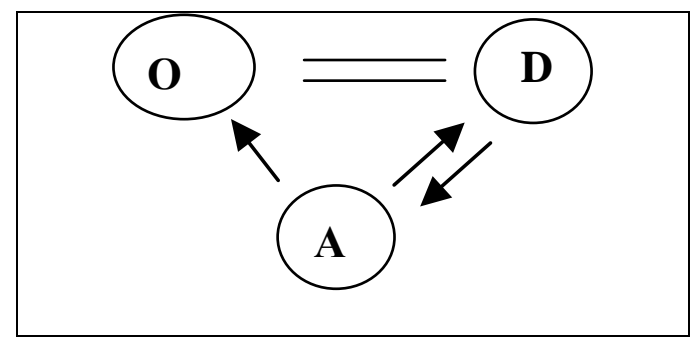

Figure 1. Empirical mode of interaction

I call empirical (see Figure 1) a mode of interaction between actor, diagram, and object whereby the actor allows him or herself to make a variety of operations on the diagram (measuring, looking at, drawing in), only constrained by the actual features of the physical drawing and the operational constraints of the physical instruments of interaction. The actor reads the results of those operations as properties ascribable to the object, thus converting their reading of the diagram into a discourse of the object. This mode of interaction takes the diagram as equivalent to the object, and hence as a referent of the discourse. The following example illustrates an empirical mode of interaction (the instructional experiment from which this episode was drawn is reported in Herbst 2003a).

Didi $^{2}$ and Gina have been comparing two cardstock triangles as regards to their area, and Didi has made the claim that since the base of one of them is twice as long as the base of the other one but the height of the former is half that of the latter, their areas should be equal. Gina adduces that the triangles can't be equal since one has a larger base and the other one a larger height. The teacher, Earl Sontag, suggests, "this would probably be a reasonable time to try the formula and see if it confirms Didi's conjecture." When Earl comes back to see them, Didi and Gina have measured bases and heights and calculated their areas using the formula. They report that the triangles are not equal, since one is $46.1\left(\mathrm{~cm}^{2}\right)$ but the other one is $44.6\left(\mathrm{~cm}^{2}\right)$.

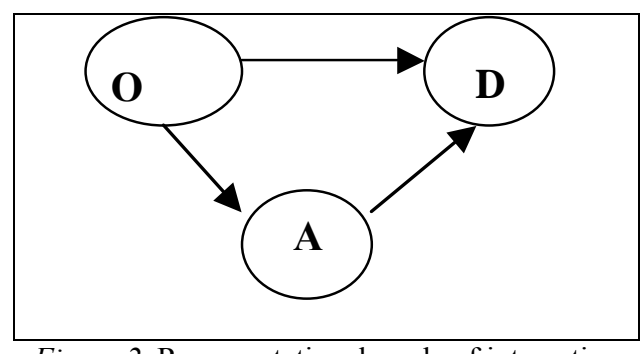

Figure 2. Representational mode of interaction

I call representational (see Figure 2) a mode of interaction between actor, diagram, and object whereby the known theoretical features of the object constrain the actor to talk in certain ways about the object and to create a diagram that is claimed to signal what is said about the object. An example of this representational interaction is in the "sequential apprehension" that Duval uses to describe the work of constructing a figure or describing a construction: "the organization of the traces does not depend on perceptual laws and cues, but on technical constraints and on mathematical properties" (p. 146). This mode of interaction takes the diagram as a one-way representation (i.e., a depiction rather than a model) of the object - the diagram is a sign for the object, which is the referent. The following example illustrates this mode of interaction.

Teacher Cecilia Marton is discussing with her students how to work a homework exercise that did not include a diagram. She asks, "is there anything that we can start with at all? Read something that you know that you can draw." Erie reads " $R S T$ is an obtuse angle with $S$ as the obtuse [angle]... [Cecilia draws such triangle on the board. Other students continue reading information from the text and Cecilia draws it on the board.] At some point in between Cecilia says, "I can already tell my drawing is a little bit, little bit not to scale, but I'm not really going to worry too much about that." Then after they have depicted what was given, Cecilia directs, "I want you to look at your drawing, because let me tell you a real common error, here, that people make, and it throws them off. A lot of people draw this so $R, S$ and $U$ are collinear.... Now, is there anything in this problem that indicates $R, S$, and $U$ should be collinear?" Students say "no" in chorus. Cecilia continues, "Nothing. If you make them collinear, you might make some mistakes with supplements or vertical angles that you shouldn't be doing, because you don't know that they're collinear." (Herbst 2003b)

Those two modes of interaction, empirical and representational, can be considered polar opposites. The empirical mode of interaction supports the hands-on geometry that students have traditionally encountered before high school, and that sustains usual activities of conjecturing based on measurements of a diagram. The representational mode of interaction, on the other hand, supports the demonstrative geometry that students have allegedly come to study in high school geometry, where the objects of study are billed as mathematical concepts defined axiomatically and whose properties are said to be proved deductively. In this "official" geometry, the diagram is only acknowledged as a depiction or an illustration. Cecilia, like many other teachers, will warn students not to trust diagrams since "they" (the textbook authors) may want to "trick" students by giving diagrams that are not up to scale. The construction of diagrams that illustrate properties taught by the teacher often builds on 
this representational mode of interaction.

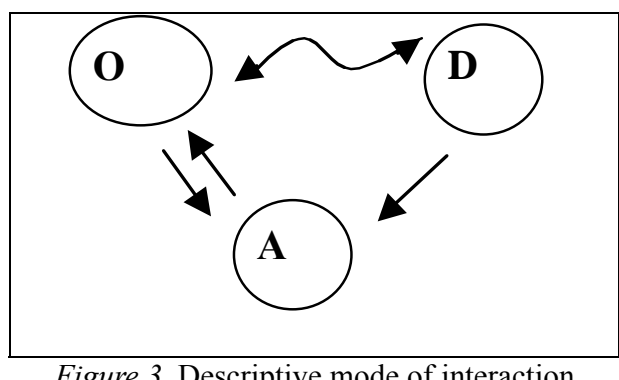

Yet, none of those modes of interaction appropriately describes the actual role that diagrams have played in making possible students' engagement in doing proofs in the United States geometry course. Our research in the history of the teaching of geometry in the US, as well as our ongoing work in geometry classrooms, suggest the need for defining a third mode of interaction. In our observations of the work that teachers do to engage students in proving we have noted how the construction of a task often involves a teacher in creating a diagram that is more than a representation of the figure alluded in the task (Herbst, 2002b). In Herbst \& Brach (2004) we use students' responses to problems presented to them as occasions to do a proof to show how students' expectation of success in doing proofs hinges on their access to a diagram with which to interact in a way that is neither empirical nor representational. From the students' perspective, this interaction involves two aspects: First, to be able to manage what to do with just what the diagram provides, without altering it; and, second, to manage to interpret all the features that are provided in the diagram, using them in writing the proof.

I call descriptive (see Figure 3) a mode of interaction between actor, object, and diagram whereby a diagram consists of two overlapping systems: a system of referents and a system of signs. ${ }^{3}$ The diagram is a particular object, which not only represents the mathematical object. To the extent that the diagram is accepted as accurate in some regards (sometimes expected to be accurate be it not for the "trickery" mentioned by Cecilia to her students), the diagram actualizes the object, making it amenable to be the source of discourse about the object or a referent for discourse, as in the empirical mode. In Figure 4, for example, the referents include a circle that is tangent to the four sides of a quadrilateral as well as several segments (the latter, notably, are not needed at all as representations of the objects mentioned in the statement of the problem). The system of signs laid on top of the diagram (through labels, hash marks, dashed lines, lettering order, and through the inclusion of otherwise irrelevant features such as auxiliary segments) moderates the extent to which the diagram can be equated to the object of such discourse. These signs point to what are things to be taken as geometric objects and how they should be taken as objects. In Figure 4, for example, the box at point $Q$ indicates that that angle is of interest and that it being a right angle is of interest. In customary situations of "doing proofs," the actor's role is to read those signs and to translate that reading into awareness of what needs to be taken as geometric object and how. The actor has to then search for properties and connections that can be ascribed to the object associated with that system of referents - at times adding more signs to record observations made. Discourse thus decodes the signs to decide which aspects of the representation can be taken as referents, describes those referents, and imputes this description to the object. The diagram in turn displays and confirms the statements produced in the discourse.

In a set of interviews where geometry students (age 14-15) were asked to comment on the likelihood of various proof problems to be assigned in their geometry class, and after having seen Figure 4 (apropos of the problem of proving that if the circle was inscribed in the quadrilateral, then $A B+C D=A C+B D$ ), Karen reacted against the possibility that the problem be given with a diagram seen previously, that did not include but the circle and the quadrilateral, criticizing the diagram: "The picture doesn't have diagonals, I mean not diagonals, radii, and those auxiliary lines drawn in, ... I wouldn't really know what to do with it unless all these lines [the ones included in the third figure] were drawn in." Commenting on Figure 4, Yuri noted: "You don't put the 90 degrees [referring to the square box marking the angle] in there unless you want people to use them...." Likewise responding to a question of which diagram would go along with a request to prove the medial line theorem, ${ }^{4}$ Hamid said: "If it was possible to solve it with either of these, I'd prefer this one because it doesn't have too much information, so it doesn't seem like we'd have to do too many steps or over complicated." (Herbst \& Brach 2004)

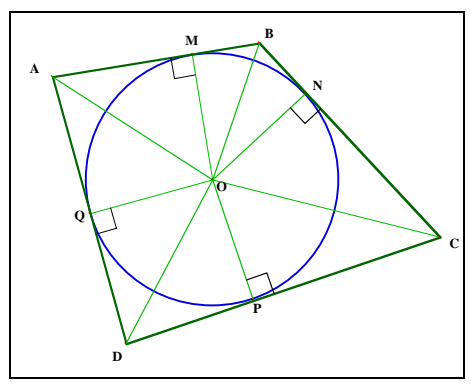

Figure 4: Third diagram used with the inscribed circle problem (in Herbst \& Brach 2004).

Such descriptive mode of interaction thus makes it possible to enhance (even ensure) students' success producing proofs. At the same time it also takes away from students some important aspects of the activity of proving, especially those connected with the reasoned building of a conjecture, or the use of argument to find out what could be true.

\section{The descriptive mode of interaction, its history, and a hypothesis about its role}

The descriptive mode of interaction is an instructionnative hybrid that combines some of the characteristics noted apropos of the empirical and representational modes. The representational mode has the geometric object known by virtue of its formal characterization as object of discourse, hence requiring the actor to reject the diagram as source of knowledge of the object and only keep it as sign. The representational mode intends to 
capture the acknowledged use of diagrams in axiomaticdeductive geometric discourse (Hilbert, 1899). This representational mode points to a norm from which usage of diagrams in the proofs done in school have been departing since the geometry class took charge of teaching students the 'art of demonstration' (and later on, 'how to do proofs' - see Herbst 2002a).

A formal proof derives new truths as discourse unfolds, from a moment where only the premises are taken as known, to a moment when also the conclusion is known. Yet, diagrams have the capacity to display at least some of those truths to be derived, in synchrony with knowledge previously sanctioned. The descriptive mode of interaction points to a particular way in which use of diagrams may depart from the representational mode. The descriptive mode of interaction points to how some of those features of a diagram, which are available to perception along with the premises of an argument (and whatever is known), can actually feed the argument being produced. Perception of a diagram thus supports and at times even replaces the logical machinery of discourse as a source of statements about the object; the system of signs not only points to what can be taken as referent, but also makes some other things invisible. The development of the descriptive mode of interaction in the US high school geometry class has followed closely the evolution of proof as an object of study.

In Herbst (2002a) I narrate how the activity of engaging students in proving acquired a stable place within the high school geometry course. I do that by tracking the evolution of students' proving from the time geometry first became a part of the high school curriculum. I describe this evolution as unfolding in three periods. A baseline period, the Era of Text, is characterized by students' reproduction of the proofs of theorems in the Euclidean canon. A transitional period, the Era of Originals, is characterized by students' engagement in proving as way to answer original questions and problems where students acquired knowledge beyond that offered by the text. A third period, the Era of Exercise, is characterized by students completion of proof-exercises that provided practice on concepts previously studied, rather than opportunities to explore new ideas. The emergence of the so-called twocolumn proof format as a standard for proof-writing is associated with this last period, in which "doing proofs" became an object of study for students. The transition along the three eras is punctuated by many other changes, both in regard to the discourse that justified the teaching of a geometry course in high school, and in regard to the mathematical activities in which students would be involved. One notable locus of those changes is the geometric diagram.

There are no records of how students produced diagrams while proving theorems during the Era of Text. But there are indications that students tended to learn the text from memory, and that such memorization was at some point during the $19^{\text {th }}$ century deemed sufficiently endemic and mathematically undesirable to trigger the inclusion of the so-called originals (Quast 1968). One can speculate that students who reproduced a diagram in its entirety before engaging in the re-production of the proof would be more likely than others (e.g., more likely than those who re-produced the diagram in synchrony with their re-production of the proof) to make mistakes in proof production or diagram construction that evidenced that they were not reasoning their way through the argument.

The originals were problems included in the geometry textbooks of early in the second half of the $19^{\text {th }}$ century that came to remedy the ills of memoristic learning. These were problems whose solution was not provided, and that gave students the opportunity to investigate on their own geometric properties that were new to them. The originals were not always accompanied by diagrams; and in the cases that they were, these diagrams contained only those objects used in the formulation of the problem, and not other objects that might be needed in the production of the proof. It was a later development in the era of originals to include so-called auxiliary constructions in the diagrams provided along with the problems. Those constructions had the purpose of suggesting what students could use in furnishing a proof. These changes in the diagram set up a different mode of interaction with the diagram, by signaling students attention to a system of referents usually more complex than that evoked in the statement of the problem, and whose existence could be taken as given, without argument (which, as mathematician Eugene Richards, 1892, p. 94, complained at the time, "may all be good classification, but it is not geometry. Neither is it good logic.") Note an example of a diagram containing one such auxiliary line $(P E)$ in Figure 5.

\section{If from a variable point $P$ in the base of an isosceles triangle $A B C$, perpendiculars, $P M, P N$ to the sides are drawn, the sum of $P M$ and $P N$ is constant, and equal to the perpendicular from $C$ upon $A B$}

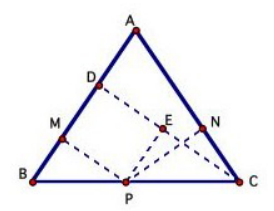

Figure 5. An "original" drawn according to an exercise from Chauvenet (1898, p.50)

During the era of exercise, the diagrams provided along with proof exercises became more and more developed, eventually including auxiliary constructions as well as conventional signs, marks on the diagram such as hash marks, arcs, numbers, and arrows (see Schultze 1912). The effect (and sometimes the explicit purpose) of those signs was to narrow down which objects, among all those made available by the diagram, was meant to be used in the proof, and how so. This practice has continued through the $20^{\text {th }}$ century. A good example of this is in the diagram provided in Figure 6, which accompanies an exercise that requires students to decide whether or not one could legitimately conclude that the triangles shown are congruent. Note that the double hash mark, used in each of the two configurations, is normally used to point to the indicated segment as being congruent to another, similarly-marked segment. But since no other segment in the configuration has two hash marks, the marking must serve another purpose. Indeed, it appears that the marks are included in the diagram to alert the student that the 
"reflexive property" (the fact that the segment is congruent to itself) will be relevant for the proof the student is to produce. ${ }^{5}$

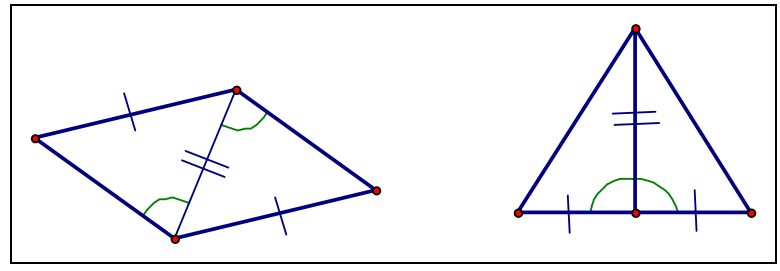

Figure 6. Two triangle congruence exercises

(drawn according to Jacobs 1974, p. 157)

The history of proving in geometry classrooms thus includes an important development in relation to students' interaction with diagrams: The emergence of a new mode of interaction with diagrams - the descriptive mode - that came to support students' accountability for "doing proofs" as well as teacher's accountability to teach them "how to do proofs." That is, the transposition of proof from being a tool of mathematical work to being an object of school learning has included making a diverse set of activities (the many proofs of many theorems) into mere instances of a generic object (proof). In the course of the $20^{\text {th }}$ century this instructional object, proof, has even received explicit definitions that attested exclusively to its formal (as opposed to substantial) characteristics. As I argue in Herbst (2002b), in order for students' productions to be reliably interpreted on behalf of such generic, formal skill, it has become necessary for instruction to create the means to convey to the student (obviously in a surreptitious way) the substance of the specific argument they need to produce. Communication of substance was needed lest the incidental unawareness of such 'ancillary' substance compromise the observation of what is 'really' at stake-deductive reasoning and clear communication. The descriptive mode of interaction explained in here has been a tool for instruction to make possible that communication of substance, hence it has come to support the expectation that students will "do proofs" (no matter what is being proved) and that such actions will attest of their learning of "proof." Partly as a result of the development of this descriptive mode of interaction, students' accountability for doing proofs, from the beginning of the era of exercise in the early $20^{\text {th }}$ century up to the present, has had a stable place in high school geometry.

I want to put forward the hypothesis that students' proving activity implicates a normative, "default" mode of interaction between student and diagram. This default is the descriptive mode. This mode of interaction has been historically constituted as a way to maintain 'doing proofs' as a situation for which students can have some responsibility, but at the expense of keeping a separation between tasks that involve deductive reasoning and tasks that involve the making of conjectures. Thus, diagrams are used to scaffold students' work when doing proof in a way that simultaneously supports students responsibility for completing a proof but also reduces students' responsibility for the argument that makes a conjecture reasonable.

This hypothesis means neither to say that all interactions between student and diagram are in fact descriptive, nor that other possible modes of interactions are in principle interdicted. Rather, the hypothesis suggests that descriptive interactions are normal, in the sense that proving tasks that build on those interactions are launched by teachers and taken on by students without any need to negotiate the didactical contract (Brousseau 1997). Conversely, the hypothesis suggests that when a task that aims at proving presumes interactions with diagrams that deviate from the descriptive norm, they $d o$ require negotiation of the didactical contract.

\section{Building reasoned conjectures: Could students take on more responsibility in proving?}

Our interview study with high school geometry students, (Herbst \& Brach 2004) complements observations of instruction in that it provides evidence that students' perceived fair share of responsibility as regards to proving occupies a rather narrow domain. Specifically, students do not expect to be responsible for producing a proof unless the conclusion to be proved, and the conditions under which that conclusion is true, are stated for them. Furthermore, the production of the diagram, which plays a key role in activating the concepts to be used in producing a proof, is, according to students, under the responsibility of the teacher (or the textbook authors). Students' responsibility when doing a proof is mainly limited to writing down steps composed of "statements" and "reasons" that connect a "given" premise to a known conclusion "to prove." Normally the statements are particular statements about a figure given; the student has to lift those statements from the diagram by appropriately interpreting the signs included. The main job of the student is to demonstrate that those statements can be connected logically to previous statements by way of reasons. Those reasons must be previously studied theorems, postulates, definitions, or the premises of the proof. The student's job is to communicate those connections clearly. Elsewhere (Herbst 2002b) I have described this as the logical ordering of a list of descriptive assertions about a figure, noting in particular how some geometric assertions - those involving incidence and separation - are often invisible; and hence very rarely will students be held accountable for proving such assertions.

The development of a descriptive mode of interaction between student and diagram has collaborated in making it possible to hold students accountable for doing those proofs. But possibly it has also collaborated in narrowing the mathematical phenomena that can be the object of students' proving activity, the role that proof plays in mathematical activity (see also Schoenfeld 1987), and the meaning of the activity of doing proofs (what doing any proof counts toward is the learning of logic and of good communication, rather than the discovery of a particular, new piece of mathematical knowledge). Such a state of affairs seems to recommend widening students' mathematical experiences, involving them in experiences in which (for example) they could use mathematical reasoning to find out new things, or in which they had to propose conditions under which something could be true. 
The possibility of creating these alternative experiences, ones that would integrate conjecturing with proving in geometry, seems to call for a different mode of interaction with diagrams.

Various studies on geometry instruction (Arzarello et al. 1998; Mariotti et al. 1997) suggest that students' meaningful construction of knowledge - and in particular their investment of mathematical reasoning and proving in such construction - is possible in the context of tasks that enable learners to act on (thus modifying) their environments. Fregona (1995) has argued that diagrams can constitute a milieu for the construction of geometric ideas. ${ }^{6}$ The notion that authentic mathematical activity involves more than merely the clear communication and logical sorting of obvious statements has prompted efforts to involve geometry students in conjecturing and problem solving-some of them in the context of using the measurement capabilities of dynamic geometry software (Hadas, Herschkovitz, and Schwartz 2000). Activities building on an empirical mode of interaction between students and diagrams have brought students closer to being able to make conjectures, yet not necessarily to having authentic experiences with the production of knowledge. Chazan (1995) has provided a critique of the traditional separation of situations for conjecturing from situations for proving. Chazan argues that to expect conjectures to emerge inductively from students' experiences, without also allowing students access to the reasons that establish a conjecture as actually true, leaves the eventual decisions on the correctness or incorrectness of the conjecture to the authority of the teacher (Christiansen, 1997, makes a related point in connection to modeling with real world data). I would like to argue that if students are to experience the production of mathematical knowledge with some agency over the means of that production, they need to be involved not just in situations of conjecturing empirically first and of proving deductively later, but on situations of making reasoned conjectures. More research needs to be done to explore what kinds of tasks can put students in contact not only with interesting phenomena, but also with the connected rationales that make those phenomena plausible. My hunch is that those tasks need to be built on neither empirical, nor representational, nor descriptive modes of interaction with diagrams, but rather on what I would like to call the generative mode of interaction.

\section{The generative mode of interaction}

The fourth mode of interaction is inspired by observations made by Netz $(1998,1999)$ apropos of the use of diagrams in Greek geometry, by Simon's (1996) argument for the need to go beyond the distinction between inductive and deductive reasoning, and also by what Duval (1995, p. 147) calls the "operative apprehension" of diagrams. Netz's (1999) study of the practices of lettering diagrams in Greek geometry allows the observation that Greek geometers would produce their diagrams at the same time that they would conceive their proofs. In other words, the diagram would not be drawn at the end to merely illustrate the written proof; nor would the diagram be drawn in its entirety before the production of the argument. Rather, the Greeks would use the argument to complexify a diagram by adding new constructions, or at least complexify the reading of a diagram by adding new signs to focus attention on previously ignored features of a diagram. In describing what he means by "operative apprehension," Duval (1995) suggests that for diagrams to work heuristically in problem solving, an individual must apprehend the diagram in an "operative" way - that is, the actor must be able to modify the diagram mentally or physically. Though it is unclear whether Duval would include what I have called above "empirical interactions" in this operative apprehension, he suggests three kinds of modifications that exemplify what I intend in generative interactions: mereologic, which includes drawing new features into the given diagram; optic, which includes applying transformations of its size or shape; and place, which includes changing location or orientation.

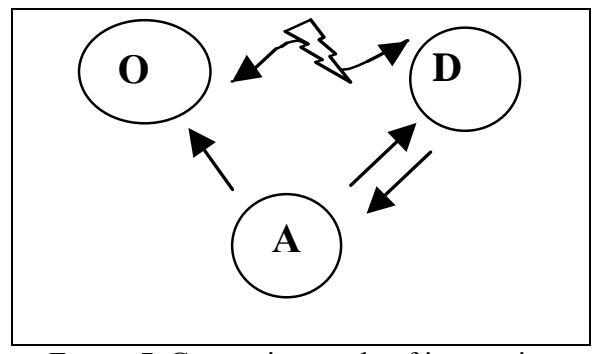

Figure 7. Generative mode of interaction

I call generative (or prescriptive; ${ }^{7}$ see Figure 6) a mode of interaction between actor, object, and diagram whereby an initial, hypothetical identification between object and diagram affords conditions and constraints for the actor to anticipate operations on the diagram and their results, and whereby the actualization of those operations on the diagram constrains the interpretation of the results of those operations in ascribing properties to the object, further differentiating the object from the diagram (which is illustrated with the lightning bolt in Figure 6). Those actions may involve creating referents (e.g., drawing in something that was not drawn originally but that is called forth in the argument the actor is making; or making an assumption or hypothesis about what something drawn is - "seeing-as", as in Jaworski 1988). Those actions may also include creating signs (e.g., new labels, new characterizations) to designate objects that were already drawn, but for which the pursuit of an argument can use a (different) way to refer to. In addition, those actions may include prescribing a way of reading a diagram so as to bypass the particularities of the diagram - thus the label prescriptive that we've used for this mode of interaction elsewhere (see Herbst 2003c). The possibility of establishing a generative mode of interaction between students and diagrams seems to be part of what is required to support students' making of reasoned conjectures - at least as it can be seen in classroom episodes in which students do use reasoning to find out what could be true, or to anticipate what has to be true, such as the following:

In Megan Keating's $10^{\text {th }}$ grade geometry class students had been working on the problem of finding a point $O$ inside a triangle so that when one connects that point with the three vertices, all 
three triangles are equal in area. Their knowledge of area at the time included how to apply formulas for area for triangles with specific dimensions, but not any of the postulates of area, which were to be introduced through this problem. ${ }^{8}$ Megan had suggested they look at how to divide a triangle into two triangles of equal area, and then try to put in a third triangle. Vito drew a triangle and the median for one of its sides (see Figure 8a) and claimed that those two triangles were equal in area. He then justified that claim, arguing that the two triangles had the same base and the same height. To make a third triangle inside the original, Vito later picked a point on the median and connected it to the two other vertices, indicating that "as long as the point $O$ was on the median, [the triangles $A O B$ and $C O B$ ] would be equal in area but not necessarily [the triangle $A O C$ ]" (see Figure $8 \mathrm{~b}$ ). To justify that statement, Brent said "If you divide the whole thing in half like we do with the other one and then you subtract the same thing from each half then you know that whatever's remaining has to be equal and so you get $\mathrm{AOB}$ is equal to BOC."9

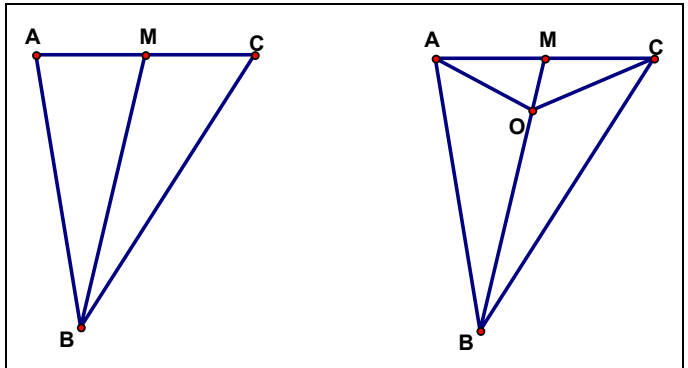

Figure $8 a$. Two triangles of equal area

$$
\text { of equal area }
$$

That particular example illustrates various aspects of the use of a diagram that make the interaction generative; and generative not just of an argument, but also of a different conception of equal area (Herbst, forthcoming a). For example, Vito picks a point $O$ on what he pretends to be (or he sees-as) the median, and draws in segments with which he makes a claim. The truth of his claim hinges on how he has generated the diagram as he works on the problem, not on the actual areas - the area-equality of two triangles hinges on picking a point on the median but does not submit to empirical verification. In Brent's case we see him strategically choosing which triangles are relevant for the argument, in spite of the fact that some of those triangles (notably $A O M$ and $C O M$ ) are not relevant for the statement of the problem. Brent also anticipates what the result of subtracting quantities should be even though it is irrelevant for him to actually compute those quantities. In drawing the diagram students don't just represent the objects involved in the argument here-their interactions with the diagram actually help them generate the argument, including the notion that $A O B$ and $B O C$ are equal in area in spite of not having a visible equal height. The diagram is not merely being described, since the students create the objects that they will talk about, and also develop ways of talking about those objects (outlining triangles, writing in numbers, using deictics) as part of a makeshift sign system. And in spite of the fact that students act on the diagram, drawing in, they don't surrender to the physical properties of the diagram - areas are equal by intellectual construction even if that intellectual construction needs physical actions to become thinkable. All of this is supported by a use of language that does not describe (e.g., verbs are not in the present tense, "is") but rather prescribe (e.g., verbs indicate necessity, "have to").

The generative mode shares with the empirical mode the capacity to feed discourse about geometric objects contingent on actions on a diagram-yet it handles those actions conceptually, bracketing the empirical reading of those actions. Discourse prescribes a reading of those actions in such a way as to generate a geometric object that is not an aggregate of experiences as much as it is an instrument to filter those experiences. On the other hand, the descriptive and generative modes of interaction with diagrams are similar in terms of what a finished diagram includes-neither just signs (as in the representational mode) nor just referents (as in the empirical mode) but a juxtaposition of both signs and referents. The generative and the descriptive mode are different, however, in terms of what actions they allow as the diagram is being modified. The descriptive mode of interaction involves very little alteration of a given diagram. These alterations include predominantly the recording on the diagram of conclusions already proved (through adding hash marks, for example) but rarely they include the drawing of auxiliary lines or the labeling of new points. Interactions that are generative of new knowledge need to allow the creation of new (or the redefinition of old) objects as they are needed to solve problems. Which brings the issue back to interaction-to the norms that regulate the situations in which students interact with diagrams, more than to the specific diagrams that are involved in those interactions.

Generative interaction points, on the one hand, to the capacity to use a representation (pictorial in this case) not just to express what one thinks, but rather as a partner to think with. On the other hand, this kind of interaction points to the capacity to use that thought to verbally repair or edit that representation, placing against the representation that exists, the geometric object that should exist instead. To interact with a diagram generatively as opposed to empirically means in particular constraining the range of things one can say about an object by way of holding oneself accountable for a conceptual reading of the actions one can take on those diagrams - or in Bachelard's words, to hold oneself accountable for showing that "the real is not what one could believe but what one should have thought" (Bachelard 1938, p. 13, my translation). To interact with a diagram generatively as opposed to descriptively also means to allow oneself to use the diagram as a model of the object - not merely as a depiction but fundamentally as a locus for the operation and source of feedback from the operations that one should expect to be able to carry out on the object itself. This is one particular feature that dynamic geometry software makes available, to varying degrees (Laborde 2000; Yerushalmy \& Chazan 1993).

However, the issue is not just to identify what sort of interaction with diagrams supports authentic intellectual activity, but also to identify the conditions that could make such activity viable in a classroom - in particular, for it to be manageable by a teacher. To this point I would like to argue that establishing and managing generative 
interactions between student, diagram and geometric object is often difficult. In the following section I provide one example of the kind of difficulties that these interactions can create for a teacher.

\section{The Circle Problem}

The following episode exemplifies what it could take to set up a generative interaction between students and diagrams, and also offers a view onto the opportunities and challenges that such an interaction presents to a teacher. One theorem about circles that teachers are expected to teach in the high school geometry course states that a circle tangent to two intersecting lines has its center on the bisector of the angle formed by the two lines, and has its points of tangency equidistant from the intersection of the two lines. What would it take to engage students in the conjecturing and proving of that theorem? In an interview with Cecilia Marton, a teacher with more than 30 years of experience teaching geometry, she indicated that for her to be able to entrust the proof of that theorem to her students, she would need to provide a diagram with all relevant elements marked, like the one on Figure 9, and state the "given" and the conclusion "to prove" explicitly. That diagram appears to be ready to engage students in interactions of the kind that I have called descriptive.

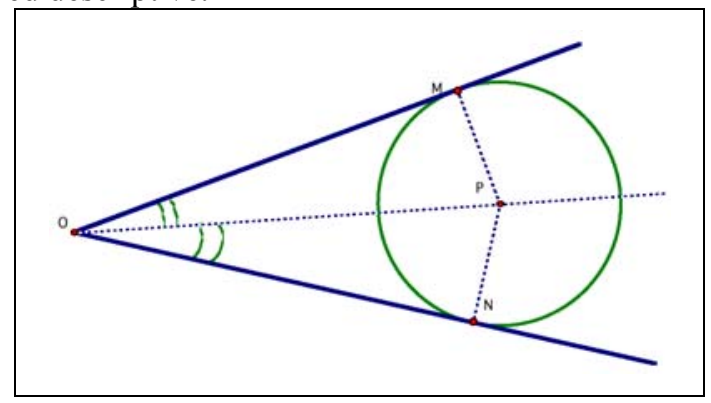

Figure 9. A diagram similar to what would be customarily used to prove the tangents theorem

In the context of the study of geometry instruction being carried out by project GRIP (Geometry, Reasoning, and Instructional Practices) we have begun to explore the question of whether the development of the tangent theorem could be shared with students by way of having them work on a problem that demanded a generative interaction between students and diagram, and what such a development would demand from a teacher. In collaboration with teacher Megan Keating we designed a lesson that was meant to engage students in formulating that tangent theorem as a way of finding out the conditions in which a problem could be solved. Students were to be given the problem stated in Figure 10, and provided with a calculator-based dynamic geometry sketch like the one in the figure, and asked to draw a circle centered on point $P$ and tangent to both lines. The lesson was taught twice in Megan's classes: first by herself, and, later in the day by a prospective teacher (James Canning) who was at the time working under Megan's supervision. A week later the problem would be used in the room of another geometry teacher, Lucille Vance.

Our analysis a priori of this task included the expectation that students would arrive at the theorem through establishing conditions for the problem to be solved. The work of finding ways to state those conditions would put them in contact with the various ideas that they would need to draw on and connect in proving the theorem. To be clear, we did not expect this would happen automatically, but that the kind of diagram and problem provided would afford the teacher a register from which to participate in a discussion that would provide context for students' making of a reasoned conjecture: A communication from the student to the teacher as to what the construction problem would be that students could be fairly expected to solve.

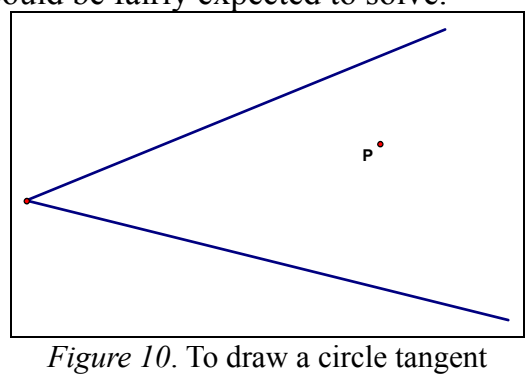
to two intersecting lines

To arrive at that situation from the given problem we expected, for example, that students might try and fit a circle in the angle and realize that it could not be done for the point given, identifying as a difficulty the fact that the purported center was not equidistant from the two legs of the angle. We expected that this would allow the teacher to engage students in rewriting the task, asking, for example, "what would you have to say about $P$ to ensure that it would be equidistant from the two legs?" or in providing directions for a diagram that would permit the construction. Students might produce responses of two kinds. Some might impose conditions that limited where to choose $P$, for example by saying that $P$ should be on the angle bisector. Others might provide specific procedures to choose one point $P$, for example by suggesting that one drew a segment joining the two legs and then found the midpoint of that segment (see Figure 11). The teacher could follow up on each of those with demands of an argument. To the first group the teacher could ask how they could make sure a point on the angle bisector is equidistant from the legs, and then what would they have to do to find out the radius of the tangent circle. To the second group the teacher could ask whether such procedure could be done with any segment whose endpoints were on the two legs of the angle. We thus expected that the discourse could foreground the connections between on the one hand the need for the center of the circle to be equidistant from the legs, made apparent by the choice of an initial point that did not have such property, and on the other hand the characteristics of a figure for which such point was equidistant from the legs.

That analysis a priori only anticipates the mathematical features of an interaction that, by virtue of taking place in a school classroom, contained other elements that had to be taken into consideration. These have to do with the customary ways in which construction problems based on a given diagram find a place in Megan's and Lucille's 
classes - customs that we presume are not particular to their classrooms as much as they are typical of high school geometry in the US (Balacheff 1999). To understand how they matter, let's see how the problem was addressed by one group of students in the first class where Megan posed the problem.

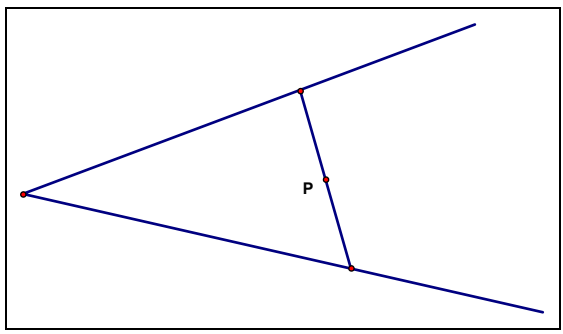

Figure 11. A possible conjecture of a better center for the tangent circle

After about 10 minutes working on the problem, the group composed of Heidi, Jessica, Mitchell, and Neil has not yet made any conjecture. This frustrates the girls. Heidi says to Megan that they are delaying because Mitchell "is getting it exactly right." Mitchell complains, "It won't give me the distance to the ray." And after trying he says, "Okay, I can't do it, man ... I just measured it, and it's definitely not working." The stakes for this group get higher when Megan asks the class who has a conjecture and all other groups bid for sharing. Megan calls Jacob, from another group to show what he has done and asks of this group "you have nothing?" Neil, who has been peripheral to the work thus far asks his group mates, "What do we have to do?" and Heidi restates the task as to "make a circle with center $P$ so that it touches those [rays]." Visibly nervous Mitchell tries repeatedly to get Megan's attention and finally asks his question "Do we have to use the point $P$ they gave us, or..." Megan answers that they "can move it." Evidently relieved Mitchell says, "Okay, we can, okay, because it didn't work where I had it.”

Mitchell's reaction shows that the task brought him to the realization that what is important for the problem to be solvable is equidistance. We are interested in his reaction not just as an adaptation to the task but also as a perturbation to the work of his teacher. In expressing relief at the possibility to move the point, Mitchell made the point to his teacher that something was odd in the way the task itself had been posed. Mitchell's initial difficulty with the problem conveyed the sense that the diagram was in some way an unfair way of engaging students in a problem. Evidence that this was a perturbation was visible later that day, immediately after James Canning presented the problem to the students in $7^{\text {th }}$ period. Before students started working on the problem, Megan made a preemptive clarification that substantially altered the mathematical nature of the task and that evidently responded to Mitchell's reaction earlier that day.

Megan: "Okay, I'm gonna say something because second hour when we did this there were a few questions. People said and uh if you're in CE1 [the name of the sketch they were to use], you see that point now that's sort of floating around between the angle...? Um, they said well, can I move that point, or do I have to leave it there? You can move anything, that you, well, some stuff you can't move on there. But you can definitely move $P$. So if you need to move $P$ to satisfy this, fine, but then the reason you moved it and where you moved it to should be part of your conjecture. So, I don't have a problem with you moving
$P$ around to get what your trying to do, is in that angle, get a circle that's tangent to both sides. You need to move $P$ around to do that, that's fine, but that better end up being part of your conjecture."

Megan's transformation of the task in this second time around is more than just a personal matter-it speaks of the extent to which it is normal in geometry classrooms to expect that students will add conditions to elements given in a diagram. The way in which Lucille Vance assigned this same task is a symptom of that constraint. When Lucille planned to teach the lesson in her class, using dynamic geometry software available on laptops, she preferred not to provide students with any pre-drawn sketch where to construct but rather to assign them the job of constructing intersecting lines and then plotting the point and constructing the tangent circle. It was far easier for her students to plot a point in a place where it looked like the construction would be possible even though they would only describe that location as "in the middle." Lucille was able to funnel that description into a reference to the angle bisector, even though the key idea that the angle bisector is important because it is the locus of points equidistant to the sides remained untouched.

There is more than students' mathematical thinking and teacher support to that thinking at play in those episodes - or perhaps, there is that, but framed in the context of student and teacher perceptions of the instructional situations in which such thinking is taking place. When students are given a task they are given it in the context of an instructional situation that makes room for the task and also points to how the doing of that task will "trade" toward the fulfillment of the didactical contract (Herbst, forthcoming b; Herbst \& Brach 2004). "Doing proofs" is one such instructional situation; doing constructions problems on given diagrams is another one; making conjectures based on empirical interactions, yet another. Whereas all of those rely on students' interactions with diagrams, none of them customarily engages students in the kind of generative interaction called forth by this circle problem, in which students had to make the choice of imposing conditions on the given point to make the problem solvable at the expense of admitting that the given problem could not be solved in general.

Reactions like that of Mitchell seem to be a normal response to the expectation that students should work with the diagram provided, rather than reject it and generate a different one that fits the problem that they could solve. One can see how doing the latter involves the student in building a reasoned conjecture: Specifically, that for a circle tangent to two intersecting lines to be constructible, its center must be equidistant from the two lines. But in order for the student to bring that issue on his or her own, to make that a deliberate move against an antagonist milieu, the task should not be one that presumes the existence of a locus for the center but one that makes no such assumption. This in turn puts the teacher in a difficult position. First of all, the teacher has to expect the students to work with the given diagram, not to discard it completely. (For example, in a later interview with Megan, she expressed dissatisfaction at the thought that students might comply with the task by 
dragging the legs of the angle so that the point $P$ would appear to be closer to the center or so that a circle would appear to be tangent.) Second, the teacher has to expect students will modify the diagram. The feedback from the given diagram to Mitchell's failed attempts is indeed ambiguous: It says to him that the actual construction is not possible and it also says where a good center should be. Students' capacity to see the second piece of information as relevant to what they are asked to do is at risk of being invisible because they are being asked to do something with that diagram not with another one. Students' chances to be involved in building a reasoned conjecture about where the center of the circle should be seem boosted by this version of the task-with the point plotted away from the angle bisector. Yet the customary situations in which students make constructions are not those where students interact generatively with diagrams (as needed in this task). Pulling off this kind of work in the class seems to require not only posing a problem and managing a discussion about how students solved the problem, but also actually negotiating the shares of accountability for the instructional situation in which they think they are. This helps understand why Megan in her later class and Lucille in her own class modified the problem in the way they did-defaulting to an empirical mode of interaction. Lucille's radical changes to the task enabled her not to have to negotiate who is accountable for doing what in the instructional situation that made room for the problem. Students thus made the conjecture that the center should be "in the middle", but specifying what "in the middle" actually means came not so much as an operative apprehension as it came as the designation of a perceptual feature of the diagram.

\section{Conclusion}

The work of "deductive guessing" — building reasoned conjectures or using deductive reasoning to find out what could or should be true - can be aided by tasks that engage students in generative interactions with diagrams. However, these interactions are not customary in geometry classes. Students' following of procedures for the construction of figures tend to engage them in a mode of interaction that I have called representational. The making of conjectures has found its way into the geometry course through engaging students in interactions with diagrams that fit the mode I have called empirical. And students' accountability for doing proofs has rested on the development of a mode of interaction that I call descriptive. Engaging students in generative interactions with diagrams thus requires a teacher to do more than design tasks and manage students' thinking in those tasks. It requires negotiating the norms of the instructional situation in which those tasks are posed. As this negotiation concerns the role of diagrams, a teacher faces the need to confront an apparent paradox that is illustrated with the circle problem - the diagram that she

\section{References}

Arzarello, F., Micheletti, C., Olivero, F., Robutti, O., and Paola, D. (1998): A model for analysing the transition to formal gives along with a problem is not necessarily the one that she expects students will use to solve the problem. Rather, that diagram is a counterpart that students will interact with in the process of exploring the conditions under which a version of the problem can be solved. Whereas the example discussed shows that students can engage in such intellectual work of thinking about a problem while interacting generatively with a diagram, it also demonstrates that in the effort to sustain a continuous engagement and avoid breaches of the didactical contract a teacher may shape that interaction with diagrams toward more customary modes.

${ }^{1}$ Research reported in this article has been done with the
support of the National Science Foundation, Grant REC
0133619 to the author. Opinions expressed are the sole responsibility of the author and do not reflect the views of the Foundation. Discussions with Maria Hamlin and other members of project GRIP (Geometry, Reasoning, and Instructional Practices) at the University of Michigan have been vital in developing these ideas. A preliminary version of this article was presented as a short oral communication at the $27^{\text {th }}$ Annual Meeting of the International Group for the Psychology of Mathematics Education, Honolulu, July 2003. I acknowledge comments from Michael Weiss and Vilma Mesa to a previous draft.

${ }^{2}$ All names used to designate students and teachers are pseudonyms.

${ }^{3}$ I use sign as the pointer to something else, referent as what the sign points to. In the empirical mode the diagram is fundamentally a referent. If anything, the role of signs is only to name: If two segments had an equal number of hash marks but were actually of different length when measured, the marks would be deemed wrongly placed. In the representational mode the diagram is a sign for a referent that exists as an abstract object manifested in discourse. In the descriptive mode at times the same graphic object can be sign and referent - two segments of actually different length can stand as referents for incidence issues (e.g., they can be taken as having a common endpoint even if that is not said) but only as signs for congruence issues (e.g., to the extent that they have the same number of hash marks they are to represent congruent segments).

${ }^{4}$ I designate by "the medial line theorem" the proposition that asserts that a segment whose endpoints are the midpoints of two sides of a triangle is parallel to the third side, and half as long.

${ }^{5}$ I thank Michael Weiss for pointing to this particular case of how diagrams are used to suggest the argument and Wendy Aaron for locating this specific example.

${ }^{6}$ By milieu it is meant a system antagonistic to the cognitive subject, in a game in which moves by the subject become more and more mathematically intelligent as they incorporate the feedback provided by the milieu (Brousseau, 1997).

${ }^{7}$ The words heuristic (Duval, 1995), transformational (Simon, 1996), and scriptive (Mason, personal communication) might also fit this mode of interaction.

${ }^{8}$ See Herbst (forthcoming a, forthcoming b).

${ }^{9}$ I thank Maria Hamlin for locating and providing a preliminary analysis of these examples.

proofs in geometry. - In: A. Olivier \& K. Newstead (Eds.), Proceedings PME 22 (v. 2). Stellenbosch, South Africa, pp. 24-31.

Bachelard, G. (1938): La formation de l'esprit scientifique. - 
Paris: Vrin.

Balacheff, N. (1999): Contract and custom: Two registers of didactical interactions. - In: The Mathematics Educator, 9, 23-29.

Brousseau, G. (1997), Theory of didactical situations in mathematics: Didactique des Mathematiques 1970-1990 (N. Balacheff, M. Cooper, R. Sutherland, and V. Warfield, Eds. and Trans.). - Dordrecht, The Netherlands: Kluwer.

Chauvenet, W. (1898): Treatise on elementary geometry (Revised and abridged by W. Byerly). Cambridge, MA: Lippincott. (Original revision published in 1887)

Chazan, D. (1995): Where do student conjectures come from? Empirical exploration in mathematics classes. National Center for Research on Teacher Learning, Craft Paper 95-8. E. Lansing, MI: Michigan State University.

Christiansen, I. (1997): When negotiation of meaning is also negotiation of task. Educational Studies in Mathematics, 34, $1-25$.

Duval, R. (1995): Geometrical pictures: Kinds of representation and specific processings. In R. Sutherland and J. Mason (Eds.), Exploiting mental imagery with computers in mathematics education (pp. 142-157). Berlin: Springer.

Fregona, D. (1995): Les figures planes comme "milieu" dans l'enseignement de la geometrie: interactions, contrats et transpositions didactiques. Thesis, Université Bordeaux I.

Hadas, N., Herschkovitz, R., and Schwartz, B. (2000): The role of contradiction and uncertainty in promoting the need to prove in Dynamic Geometry environments. Educational Studies in Mathematics, 44, 127-150.

Herbst, P. (2002a): Establishing a custom of proving in American school geometry: evolution of the two-column proof in the early twentieth century. Educational Studies in Mathematics, 49, 283-312.

Herbst, P. (2002b): Engaging students in proving: A double bind on the teacher. Journal for Research in Mathematics Education, 33, 176-203

Herbst, P. (2003a): Using novel tasks in teaching mathematics: Three tensions affecting the work of the teacher. American Educational Research Journal, 40, 197-238.

Herbst, P. (2003b): Geometry, reasoning, and instructional practices. Unpublished raw data. University of Michigan, Ann Arbor.

Herbst, P. (2003c): Descriptive and prescriptive interactions with diagrams and customary situations of proving in geometry. In N. Pateman, B. Dougherty, and J. Zilliox (Eds.), Proceedings of the 2003 Joint Meeting of PME and PMENA (Vol 1, p. 229). Honolulu: University of Hawaii.

Herbst, P. and Brach, C. (2004): Proving and proof in high school geometry: What is 'it' that is going on for students and how do they make sense of it? Paper presented at the Annual Meeting of the American Educational Research Association, San Diego, California.

Herbst, P. (forthcoming a): Conceptualizing 'equal area' while proving a claim about equal areas. In revision for Recherches en Didactique des Mathématiques.

Herbst, P. (forthcoming b): Building reasoned conjectures while working on problems: Tensions in teaching geometry. Accepted at Journal for Research in Mathematics Education.

Hilbert, D. (1971): Foundations of Geometry. (L. Unger, Trans., P. Bernays, Rev.). Open Court, La Salle, IL. (Original work published in German in 1899)

Jacobs, H. (1974): Geometry. San Francisco: W. H. Freeman and company.

Jaworski, B. (1988): 'Is' versus 'seeing as': Constructivism and the mathematics classroom. In D. Pimm (Ed.), Mathematics, Teachers and Children (pp. 287-296). London: Hodder \& Stoughton.

Laborde, C. (2000): Dynamic geometry environments as a source of rich learning contexts for the complex activity of proving. Educational Studies in Mathematics, 44, 151-161.

Lakatos, I. (1976): Proofs and refutations: The logic of mathematical discovery. Cambridge University Press.

Mariotti, M. A., Bartolini-Bussi, M., Boero, P., Ferri, F. and Garuti, R. (1997): Approaching geometry theorems in contexts: From history and epistemology to cognition. In E. Pehkonen (Ed.), Proc. PME 21 (v. 1, pp. 180-195). Lahti, Finland.

Moise, E. (1975): The meaning of Euclidean geometry in school mathematics. The Mathematics Teacher, 68, 472-477

Netz, R. (1998): Greek mathematics diagrams: their use and their meaning. For the Learning of Mathematics, 18(3), 33-39.

Netz, R. (1999): The shaping of deduction in Greek mathematics. Cambridge: Cambridge University Press.

Quast, W.G. (1968): Geometry in the High Schools of the United States: An Historical Analysis from 1890 to 1966. Unpublished doctoral dissertation. Rutgers - The State University of New Jersey, New Brunswick.

Richards, E. (1892): Old and new methods in elementary geometry. Educational Review, 3, 31-39.

Schoenfeld, A. (1987): On having and using geometrical knowledge. In J. Hiebert (Ed.), Conceptual and Procedural Knowledge: The case of mathematics (pp. 225-264). Hillsdale, NJ: Erlbaum

Schultze, A.: 1912, The Teaching of Mathematics in Secondary Schools, MacMillan, New York.

Simon, M. (1996): Beyond inductive and deductive reasoning: The search for a sense of knowing. Educational Studies in Mathematics, 30, 197-210.

Usiskin, Z. (1980): What should not be in the algebra and geometry curricula of average college-bound students? The Mathematics Teacher, 73, 413-424.

Yerushalmy, M. and Chazan, D. (1993): Overcoming visual obstacles with the aid of the Supposer. In J. Schwartz, M. Yerushalmy, and B. Wilson (Eds.), The Geometric Supposer: What is it a case of? (pp. 25-56). Hillsdale, NJ: Erlbaum.

\section{Author}

Patricio Herbst

School of Education, University of Michigan

610 East University Avenue, \#1302C

Ann Arbor, MI 48109-1259

U.S.A.

pgherbst@umich.edu 